\title{
Solution of the Differential-Difference Equations by Optimal Homotopy Asymptotic Method
}

\author{
H. Ullah, S. Islam, M. Idrees, and M. Fiza \\ Department of Mathematics, Abdul Wali Khan University, Mardan 23200, Pakistan \\ Correspondence should be addressed to H. Ullah; hakeemullah1@gmail.com
}

Received 20 January 2014; Revised 1 May 2014; Accepted 1 May 2014; Published 29 May 2014

Academic Editor: Fuding Xie

Copyright (C) $2014 \mathrm{H}$. Ullah et al. This is an open access article distributed under the Creative Commons Attribution License, which permits unrestricted use, distribution, and reproduction in any medium, provided the original work is properly cited.

We applied a new analytic approximate technique, optimal homotopy asymptotic method (OHAM), for treatment of differentialdifference equations (DDEs). To see the efficiency and reliability of the method, we consider Volterra equation in different form. It provides us with a convenient way to control the convergence of approximate solutions when it is compared with other methods of solution found in the literature. The obtained solutions show that OHAM is effective, simpler, easier, and explicit.

\section{Introduction}

In physical science the DDEs play a vital role in modeling of the complex physical phenomena. The DDEs models are used in vibration of particles in lattices, the flow of current in a network, and the pulses in biological chains. The models containing DDEs have been investigated by numerical techniques such as discretizations in solid state physics and quantum mechanics. In the last decades most of the research work has been done on DDEs. Levi and Yamilov $[1,2]$ attribute their work to classification of DDEs and connection of integrable partial differential equation (PDEs) and DDEs [3, 4]. In [5-8] the exact solutions of the DDEs have been studied. For the solution of the DDEs, Zou et al. extended the homotopy analysis method (HAM) $[9,10]$. Wang et al. extended Adomian decomposition method (ADM) for solving nonlinear difference-differential equations (NDDEs) and got a good accuracy with the analytic solution [11]. Recently, Marinca et al. introduced optimal homotopy asymptotic method (OHAM) [12-16] for the solution of nonlinear problems. The validity of OHAM is independent of whether or not the nonlinear problems contain small parameters.

The motivation of this paper is to extend OHAM for the solution of NDDEs. In [17-20] OHAM has been proved to be useful for obtaining an approximate solution of nonlinear boundary value problems of differential equations. In this work, we have proved that OHAM is also useful and reliable for the solution of NDDEs, hence showing its validity and great potential for the solution of NDDEs phenomenon in science and engineering.

In the succeeding section, the basic idea of OHAM [1216] is formulated for the solution of boundary value problems. In Section 3, the effectiveness of the modified formulation of OHAM for NDDEs has been studied.

\section{Basic Mathematical Theory of OHAM for NDDE}

Let us take OHAM to the following differential-difference equation:

$$
\mathscr{A}\left(v_{n}(t)\right)+f(t)=0, \quad n \in \Omega,
$$

with boundary conditions:

$$
\mathscr{B}\left(v_{n}(t), \frac{\partial v_{n}(t)}{\partial t}\right)=0 . \quad n \in \Gamma,
$$

where $\mathscr{A}$ is a differential operator, $v_{n}(t)$ is an unknown function, $n$ and $t$ denote spatial and temporal independent variables, respectively, $\Gamma$ is the boundary of $\Omega$, and $f_{n}(t)$ is a known analytic function. $\mathscr{A}$ can be divided into two parts $\mathscr{L}$ and $\mathscr{N}$ such that

$$
\mathscr{A}=\mathscr{L}+\mathcal{N},
$$


where $\mathscr{L}$ is simpler part of the partial differential equation which is easier to solve and $\mathscr{N}$ contains the remaining part of $\mathscr{A}$. So (1) can be written as

$$
\mathscr{L}\left(v_{n}(t)\right)+f(t)+\mathcal{N}\left(v_{n}(t), v_{n-k}(t), v_{n+k}(t)\right)=0 .
$$

According to OHAM, one can construct an optimal homotopy $\phi_{n}(t, q): \Omega \times[0,1] \rightarrow \Re$ satisfying

$$
\begin{aligned}
&(1-q)\left[\mathscr{L}\left(\phi_{n}(t, q)\right)+f(t)\right] \\
&=H(q)[ \mathscr{L}\left(\phi_{n}(t, q)\right)+f(t) \\
&\left.+\mathcal{N}\left(\phi_{n}(t, q), \phi_{n-k}(t, q), \phi_{n+k}(t, q)\right)\right], \\
& \mathscr{B}\left(\phi_{n}(t, q), \frac{\partial \phi_{n}(t, q)}{\partial t}\right)=0,
\end{aligned}
$$

where $q \in[0,1]$ is an embedding parameter, $\phi_{n}(t, q)$ is an unknown function, and $H(q)$ is a nonzero auxiliary function. The auxiliary function $H(q)$ is nonzero for $q \neq 0$ and $H(0)=$ 0 . Equation (5) is the structure of OHAM homotopy. Clearly we have

$$
\begin{aligned}
q & =0 \Longrightarrow H\left(\phi_{n}(t ; 0), 0\right) \\
& =\mathscr{L}\left(\phi_{n}(t ; 0)\right)+f(t)=0, \\
q & =1 \Longrightarrow H\left(\phi_{n}(t ; 1), 1\right) \\
& =H(1)\left\{\mathscr{A}\left(\phi_{n}(t ; 1)\right)+f(t)\right\}=0 .
\end{aligned}
$$

Obviously, when $q=0$ and $q=1$, we obtain

$$
\begin{aligned}
& q=0 \Longrightarrow \phi_{n}(t, 0)=v_{n 0}(t), \\
& q=1 \Longrightarrow \phi_{n}(t, 1)=v_{n}(t),
\end{aligned}
$$

respectively. Thus, as $q$ varies from 0 to 1 , the solution $\phi_{n}(t, q)$ varies from $v_{n 0}(t)$ to $v_{n}(t)$, where $v_{n 0}(t)$ is the zeroth order solution and can be obtained from (10).

Expanding $\phi_{n}\left(t, q, C_{i}\right), \phi_{n-k}\left(t, q, C_{i}\right), \phi_{n+k}\left(t, q, C_{i}\right)$ by Taylors series and choosing $H(q)$ as given

$$
\begin{aligned}
H(q)=q C_{1}+ & q^{2} C_{2}+q^{3} C_{3}+\cdots+q^{m} C_{m}, \\
\phi_{n-k}\left(t, q, C_{i}\right)= & v_{(n-k) 0}(t) \\
& +\sum_{m=1}^{\infty} v_{(n-k) m}\left(t, C_{i}\right) q^{m}, \\
\phi_{n}\left(t, q, C_{i}\right)= & v_{(n) 0}(t) \\
& +\sum_{m=1}^{\infty} v_{(n) m}\left(t, C_{i}\right) q^{m}, \\
\phi_{n+k}\left(t, q, C_{i}\right)= & v_{(n+k) 0}(t) \\
& +\sum_{m=1}^{\infty} v_{(n+k) m}\left(t, C_{i}\right) q^{m},
\end{aligned}
$$

where $C_{i}, i=1,2,3, \ldots$ are constants and to be determined and $m \in N$.
Now substituting (9) into (5) and equating the coefficient of like powers of $q$, we obtain

$$
\begin{aligned}
& \mathscr{L}\left(v_{n 0}(t)\right)+f(t)=0, \quad \mathscr{B}\left(v_{n 0}(t), \frac{\partial v_{n 0}(t)}{\partial t}\right)=0, \\
& \mathscr{L}\left(v_{n 1}(t)\right)-\mathscr{L}\left(v_{n 0}(t)\right) \\
& =C_{1}\left[\mathscr{L}\left(v_{n 0}(t)\right)+\mathcal{N}\left(v_{(n-k) 0}(t), v_{n 0}(t), v_{(n+k) 0}(t)\right)\right], \\
& \mathscr{B}\left(v_{n 1}(t), \frac{\partial v_{n 1}(t)}{\partial t}\right)=0, \\
& \mathscr{L}\left(v_{n 2}(t)\right)-\mathscr{L}\left(v_{n 1}(t)\right) \\
& =C_{1}\left[\mathscr{L}\left(v_{n 1}(t)\right)+\mathcal{N}\left(v_{(n-k) 1}(t), v_{n 1}(t), v_{(n+k) 1}(t)\right)\right] \\
& \quad+C_{2}\left[\mathscr{L}\left(v_{n 0}(t)\right)+\mathcal{N}\left(v_{(n-k) 0}(t), v_{n 0}(t), v_{(n+k) 0}(t)\right)\right], \\
& \mathscr{B}\left(u_{n 2}(n, t), \frac{\partial u_{n 2}(n, t)}{\partial t}\right)=0, \\
& \mathscr{L}\left(v_{n 3}(t)\right)-\mathscr{L}\left(v_{n 2}(t)\right) \\
& =C_{1}\left[\mathscr{L}\left(v_{n 2}(t)\right)+\mathcal{N}\left(v_{(n-k) 2}(t), v_{n 2}(t), v_{(n+k) 2}(t)\right)\right] \\
& \quad+C_{2}\left[\mathscr{L}\left(v_{n 1}(t)\right)+\mathcal{N}\left(v_{(n-k) 1}(t), v_{n 1}(t), v_{(n+k) 1}(t)\right)\right] \\
& \quad+C_{3}\left[\mathscr{L}\left(v_{n 0}(t)\right)+\mathcal{N}\left(v_{(n-k) 0}(t), v_{n 0}(t), v_{(n+k) 0}(t)\right)\right], \\
& \mathscr{B}\left(v_{n 3}(t), \frac{\partial v_{n 3}(t)}{\partial t}\right)=0, \\
& \quad=\sum_{i=1}^{m} \sum_{j=m-1}^{0} C_{i}\left[\mathscr{L}\left(v_{n j}(t)\right)\right. \\
& \mathscr{L}\left(v_{n j}(t)\right)-\mathscr{L}\left(v_{n(j-1)}(t)\right) \\
& \quad \vdots \\
&
\end{aligned}
$$

We obtained the zeroth order solution and first, second, third, and the general order solutions by solving (10)-(14). In the same way the remaining solutions can be determined. It has been observed that the convergence of the series (9) depends on the auxiliary constants $C_{i}$. If it is convergent at $q=1$, one has

$$
v_{n}\left(t ; C_{i}\right)=v_{n 0}(x, t)+\sum_{k \geq 1} v_{n k}\left(t ; C_{i}\right) .
$$

Substituting (15) into (1), it results in the following expression for residual:

$$
R\left(t, C_{i}\right)=\mathscr{L}\left(\widetilde{v}_{n}\left(t, C_{i}\right)\right)+f(t)+\mathcal{N}\left(\widetilde{v}_{n}\left(t, C_{i}\right)\right) .
$$


If $R\left(t, C_{i}\right)=0$, then $\widetilde{v}_{n}\left(t, C_{i}\right)$ is the exact solution of the problem. Generally it does not happen, especially in nonlinear problems.

For the determinations of auxiliary constants, $C_{i}, i=$ $1,2,3, \ldots$, there are different methods like Galerkin's method, Ritz method, least squares method, and collocation method. One can apply the method of least squares as follows:

$$
J\left(C_{i}\right)=\int_{a}^{b} \int_{\Omega} R^{2}\left(t, C_{i}\right) d n d t,
$$

where $a$ and $b$ are two values, depending on the nature of the given problem.

The auxiliary constants $C_{i}$ can be optimally calculated as

$$
\frac{\partial J}{\partial C_{1}}=\frac{\partial J}{\partial C_{2}}=\frac{\partial J}{\partial C_{3}} \cdots \frac{\partial J}{\partial C_{m}}=0
$$

The $m$ th order approximate solution can be obtained by these obtained constants.

The convergence of OHAM is directly proportional to the number of optimal constants $C_{i}$.

\section{Application of OHAM to Difference-Differential Equations}

To show the validity and effectiveness of OHAM formulation to difference-differential equation, we apply it to Voltera equations in different form.

Model 1. We consider Voltera equation of the form

$$
\frac{\partial v_{n}(t)}{\partial t}=v_{n}(t)\left(v_{n-1}(t)-v_{n+1}(t)\right), \quad n>0, t \in[0,1],
$$

with initial conditions

$$
v_{n}(0)=n .
$$

The exact solution of (3.1) is given by

$$
v_{n}(t)=\frac{n}{1+n t},
$$

and we take

$$
\begin{aligned}
v_{n}(t)= & v_{n 0}(t)+p v_{n 1}(t)+p^{2} v_{n 2}(t)+p^{3} v_{n 3}(t), \\
v_{n+1}(t)= & v_{(n+1) 0}(t)+p v_{(n+1) 1}(t) \\
& +p^{2} v_{(n+1) 2}(t)+p^{3} v_{(n+1) 3}(t) \\
v_{n-1}(t)= & v_{(n-1) 0}(t)+p v_{(n-1) 1}(t) \\
& +p^{2} v_{(n-1) 2}(t)+p^{3} v_{(n-1) 3}(t) .
\end{aligned}
$$

According to (1), we have

$$
\begin{aligned}
& \mathscr{L}=\frac{\partial v_{n}(t)}{\partial t}, \quad f(t)=0, \\
& \mathcal{N}=-v_{n}(t)\left(v_{n-1}(t)-v_{n+1}(t)\right) .
\end{aligned}
$$

Using (19), (20), and (22) into (5) and using the method discussed in Section 2 lead to the following.

Zeroth Order Problem. Consider

$$
\frac{\partial v_{n 0}(t)}{\partial t}=0
$$

with

$$
v_{n 0}(0)=n,
$$

from which we obtain

$$
v_{n 0}(t)=n .
$$

First Order Problem. One has

$$
\begin{aligned}
\frac{\partial v_{n 1}(t)}{\partial t}= & \left(1+C_{1}\right) \frac{\partial v_{n 0}(t)}{\partial t} \\
& +C_{1}\left(v_{(n+1) 0}(t)-v_{(n-1) 0}(t)\right) v_{n 0}(t),
\end{aligned}
$$

with

$$
v_{n 1}(0)=0 \text {. }
$$

Its solution is

$$
v_{n 1}(t)=2 C_{1} n t
$$

Second Order Problem. Consider

$$
\begin{aligned}
\frac{\partial v_{n 2}(t)}{\partial t}= & \left(1+C_{1}\right) \frac{\partial v_{n 1}(t)}{\partial t}+C_{2} \frac{\partial v_{n 0}(t)}{\partial t} \\
& +C_{1}\left(v_{(n+1) 0}(t)-v_{(n-1) 0}(t)\right) v_{n 1}(t) \\
& +C_{1}\left(v_{(n+1) 1}(t)-v_{(n-1) 1}(t)\right) v_{n 0}(t) \\
& +C_{2}\left(v_{(n+1) 0}(t)-v_{(n-1) 0}(t)\right) v_{n 0}(t),
\end{aligned}
$$

with

$$
v_{n 2}(0)=0,
$$

$$
v_{n 2}(t)=2\left(C_{1} n t+C_{1}^{2} n t+C_{2} n t+2 C_{1}^{2} n t^{2}\right) .
$$

Third Order Problem. One has

$$
\begin{aligned}
\frac{\partial v_{n 3}(t)}{\partial t}= & \left(1+C_{1}\right) \frac{\partial v_{n 2}(t)}{\partial t}+C_{2} \frac{\partial v_{n 1}(t)}{\partial t}+C_{3} \frac{\partial v_{n 0}(t)}{\partial t} \\
& +C_{1}\left(v_{(n+1) 0}(t)-v_{(n-1) 0}(t)\right) v_{n 2}(t) \\
& +C_{1}\left(v_{(n+1) 1}(t)-v_{(n-1) 1}(t)\right) v_{n 1}(t) \\
& +C_{2}\left(v_{(n+1) 0}(t)-v_{(n-1) 0}(t)\right) v_{n 1}(t) \\
& +C_{1}\left(v_{(n+1) 2}(t)-v_{(n-1) 2}(t)\right) v_{n 0} \\
& +C_{2}\left(v_{(n+1) 1}(t)-v_{(n-1) 1}(t)\right) v_{n 0}(t) \\
& +C_{3}\left(v_{(n+1) 0}(t)-v_{(n-1) 0}(t)\right) v_{n 0}(t),
\end{aligned}
$$


TABLE 1: Comparisons of OHAM, exact, and ADM results at different values of $n$ at $t=1$.

\begin{tabular}{ccccc}
\hline$n$ & OHAM & Exact & ADM & Abs error \\
\hline 1 & 0.333333 & 0.333333 & 0.333333 & $7.17103 \times 10^{-7}$ \\
2 & 0.666665 & 0.666665 & 0.666665 & $1.43421 \times 10^{-6}$ \\
3 & 0.999998 & 0.999998 & 0.999998 & $2.15131 \times 10^{-6}$ \\
4 & 1.33333 & 1.33333 & 1.33333 & $2.86841 \times 10^{-6}$ \\
5 & 1.66666 & 1.66666 & 1.66666 & $3.58551 \times 10^{-6}$ \\
6 & 2 & 2 & 2 & $4.30262 \times 10^{-6}$ \\
7 & 2.33333 & 2.33333 & 2.33333 & $5.01972 \times 10^{-6}$ \\
8 & 2.66666 & 2.66666 & 2.66666 & $5.73682 \times 10^{-6}$ \\
9 & 2.99999 & 2.99999 & 2.99999 & $6.45392 \times 10^{-6}$ \\
10 & 3.33333 & 3.33333 & 3.33333 & $7.17103 \times 10^{-6}$ \\
\hline
\end{tabular}

TABle 2: Comparisons of first, second, and third order and exact solutions at different values of $n$ at $t=1$.

\begin{tabular}{ccccc}
\hline$n$ & First order & Second order & Third order & Exact sol. \\
\hline 1 & 0.527142 & 0.858619 & 0.333333 & 0.333333 \\
2 & 1.05428 & 1.71724 & 0.666665 & 0.666665 \\
3 & 1.58143 & 2.57586 & 0.999998 & 0.999998 \\
4 & 2.10857 & 3.43447 & 1.33333 & 1.33333 \\
5 & 2.63571 & 4.29309 & 1.66666 & 1.66666 \\
6 & 3.16285 & 5.15171 & 2 & 2 \\
7 & 3.68999 & 6.01033 & 2.33333 & 2.33333 \\
8 & 4.21713 & 6.86895 & 2.66666 & 2.66666 \\
9 & 4.74428 & 7.72757 & 2.99999 & 2.99999 \\
10 & 5.27142 & 8.58619 & 3.33333 & 3.33333 \\
\hline
\end{tabular}

with

$$
\begin{gathered}
v_{n 3}(0)=0 \\
v_{n 3}(t)=\frac{2}{3}\left(3 C_{1} n t+6 C_{1}^{2} n t+3 C_{1}^{3} n t+3 C_{2} n t+3 C_{3} n t\right. \\
\left.+12 C_{1}^{2} n t^{2}+12 C_{1}^{3} n t^{2}+6 C_{1} C_{2} n t^{2}+4 C_{1}^{3} n t^{3}\right) .
\end{gathered}
$$

Adding (26), (29), (32), and (35), we obtain

$$
\begin{aligned}
v_{n}(t)= & v_{n 0}(t)+v_{n 1}\left(t, C_{1}\right)+v_{n 2}\left(t, C_{1}, C_{2}\right) \\
& +v_{n 3}\left(t, C_{1}, C_{2}, C_{3}\right), \\
v_{n}(t)= & n+2 C_{1} n t+2\left(C_{1} n t+C_{1}^{2} n t+C_{2} n t+2 C_{1}^{2} n t^{2}\right) \\
& +\frac{2}{3}\left(3 C_{1} n t+6 C_{1}^{2} n t+3 C_{1}^{3} n t+3 C_{2} n t+3 C_{3} n t\right. \\
& \left.+12 C_{1}^{2} n t^{2}+12 C_{1}^{3} n t^{2}+6 C_{1} C_{2} n t^{2}+4 C_{1}^{3} n t^{3}\right) .
\end{aligned}
$$

For the computation of the constants $C_{1}, C_{2}$, and $C_{3}$ applying the method of least square mentioned in (16)-(18), we get

$$
\begin{aligned}
& C_{1}=-0.23642908394894221, \\
& C_{2}=0.2344713680405294, \\
& C_{3}=-0.4015040325725273 \text { for } n=1 .
\end{aligned}
$$

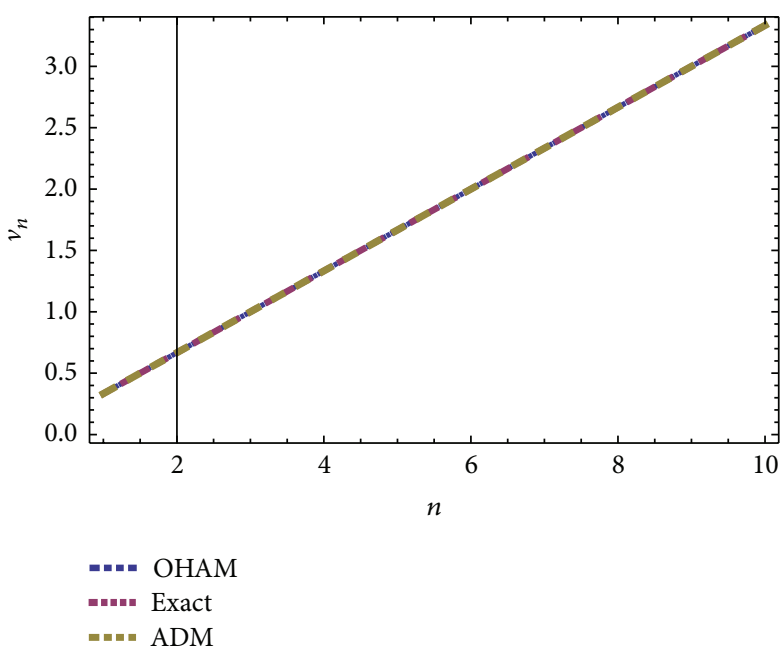

FIgUre 1: Comparison of OHAM, exact, and ADM solutions at $t=1$.

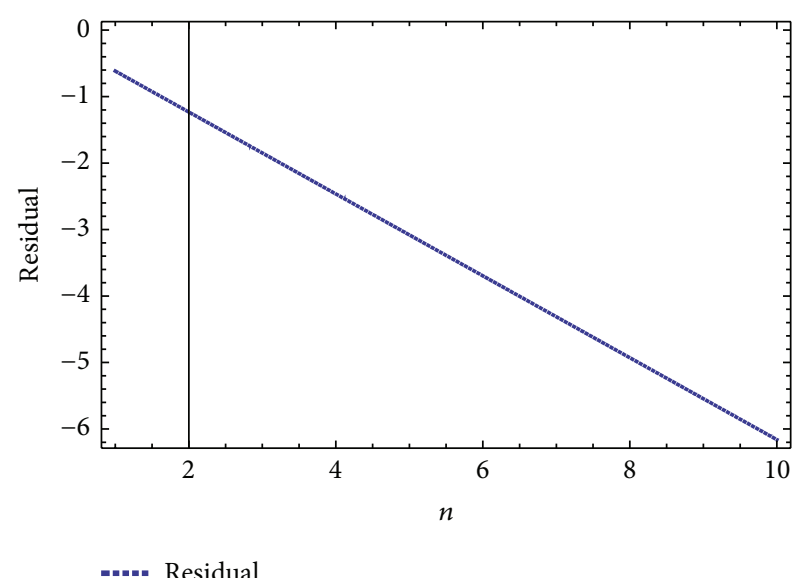

Figure 2: Residual of (19) at $t=1$.

Putting these values in (37), we obtained the approximate solution of the form

$$
\begin{aligned}
v_{n}(t)= & n-0.472858 n t+2\left(0.05 n t+0.111797 n t^{2}\right) \\
& +\frac{2}{3}\left(-0.914641 n t+0.179576 n t^{2}-0.0528643 n t^{3}\right) .
\end{aligned}
$$

The exact solution of the problem (3.1) is [11]

$$
v_{n}(t)=\frac{n}{1+n t}
$$

The Adomian solution with Padé approximation of (3.1) is given by [11]

$$
v_{n}(t)=\frac{n}{1+n t}
$$


Model 2. Now consider another form of Volterra equation

$$
\begin{aligned}
\frac{\partial v_{n}(t)}{\partial t}= & v_{n}(t)\left(v_{n-1}(t)-v_{n+1}(t)\right) \\
& +v_{n}(t)\left(v_{n-2}(t)-v_{n+2}(t)\right), \quad n>0, t \in[0,1],
\end{aligned}
$$

with initial conditions

$$
v_{n}(0)=n
$$

The exact solution of (42) is given by

$$
v_{n}(t)=\frac{n}{1+6 t} .
$$

We take

$$
\begin{aligned}
v_{n}(t)= & v_{n 0}(t)+p v_{n 1}(t)+p^{2} v_{n 2}(t) \\
& +p^{3} v_{n 3}(t), \\
v_{n+1}(t)= & v_{(n+1) 0}(t)+p v_{(n+1) 1}(t) \\
& +p^{2} v_{(n+1) 2}(t)+p^{3} v_{(n+1) 3}(t), \\
v_{n-1}(t)= & v_{(n-1) 0}(t)+p v_{(n-1) 1}(t) \\
& +p^{2} v_{(n-1) 2}(t)+p^{3} v_{(n-1) 3}(t), \\
v_{n+2}(t)= & v_{(n+2) 0}(t)+p v_{(n+2) 1}(t) \\
& +p^{2} v_{(n+2) 2}(t)+p^{3} v_{(n+2) 3}(t), \\
v_{n-2}(t)= & v_{(n-2) 0}(t)+p v_{(n-2) 1}(t) \\
& +p^{2} v_{(n-2) 2}(t)+p^{3} v_{(n-2) 3}(t) .
\end{aligned}
$$

According to (1), we have

$$
\begin{aligned}
\mathscr{L}= & \frac{\partial v_{n}(t)}{\partial t}, \quad f(t)=0, \\
\mathcal{N}= & -v_{n}(t)\left(v_{n-1}(t)-v_{n+1}(t)\right) \\
& -v_{n}(t)\left(v_{n-2}(t)-v_{n+2}(t)\right) .
\end{aligned}
$$

Using (42), (43), and (45) into (5) and using the method discussed in Section 2 lead to the following.

\section{Zeroth Order Problem. Consider}

$$
\frac{\partial v_{n 0}(t)}{\partial t}=0
$$

with

$$
v_{n 0}(0)=n,
$$

from which we obtain

$$
v_{n 0}(t)=n \text {. }
$$

First Order Problem. One has the following:

$$
\begin{aligned}
\frac{\partial v_{n 1}(t)}{\partial t}= & \left(1+C_{1}\right) \frac{\partial v_{n 0}(t)}{\partial t} \\
& +C_{1}\left(v_{(n+2) 0}(t)-C_{1} v_{(n-2) 0}(t)\right) v_{n 0}(t) \\
& +C_{1}\left(v_{(n+1) 0}(t)-C_{1} v_{(n-1) 0}(t)\right) v_{n 0}(t),
\end{aligned}
$$

with

$$
v_{n 1}(0)=0 \text {. }
$$

Its solution is

$$
v_{n 1}(t)=6 C_{1} n t
$$

Second Order Problem. Consider

$$
\begin{aligned}
\frac{\partial v_{n 2}(t)}{\partial t}= & \left(1+C_{1}\right) \frac{\partial v_{n 1}(t)}{\partial t}+C_{2} \frac{\partial v_{n 0}(t)}{\partial t} \\
& +C_{1}\left(v_{(n+2) 1}(t)-v_{(n-2) 1}(t)\right) v_{n 0}(t) \\
& +C_{2}\left(v_{(n+2) 0}(t)-v_{(n-2) 0}(t)\right) v_{n 0} \\
& +C_{1}\left(v_{(n+2) 0}(t)-v_{(n-2) 0}(t)\right) v_{n 1}(t) \\
& +C_{1}\left(v_{(n+1) 0}(t)-v_{(n-1) 0}(t)\right) v_{n 1}(t) \\
& +C_{2}\left(v_{(n+1) 0}(t)-v_{(n-1) 0}(t)\right) v_{n 0}(t) \\
& +C_{1}\left(v_{(n+1) 1}(t)-v_{(n-1) 1}(t)\right) v_{n 0}(t),
\end{aligned}
$$

with

$$
\begin{gathered}
v_{n 2}(0)=0 \\
v_{n 2}(t)=6 n t\left(C_{1}+C_{2}+C_{1}^{2}(1+6 t)\right) .
\end{gathered}
$$

Third Order Problem. One has

$$
\begin{aligned}
\frac{\partial v_{n 3}(t)}{\partial t}= & \left(1+C_{1}\right) \frac{\partial v_{n 2}(t)}{\partial t}+C_{2} \frac{\partial v_{n 1}(t)}{\partial t}+C_{3} \frac{\partial v_{n 0}(t)}{\partial t} \\
& +C_{1}\left(v_{(n+2) 2}(t)-v_{(n-2) 2}(t)\right) v_{n 0}(t) \\
& +C_{2}\left(v_{(n+2) 1}(t)-v_{(n-2) 1}(t)\right) v_{n 0}(t) \\
& +C_{3}\left(v_{(n+2) 0}(t)-v_{(n-2) 0}(t)\right) v_{n 0}(t) \\
& +C_{1}\left(v_{(n+2) 1}(t)-v_{(n-2) 1}(t)\right) v_{n 1}(t)
\end{aligned}
$$




$$
\begin{aligned}
& +C_{2}\left(v_{(n+2) 0}(t)-v_{(n-2) 0}(t)\right) v_{n 1}(t) \\
& +C_{1}\left(v_{(n+2) 0}(t)-v_{(n-2) 0}(t)\right) v_{n 2}(t) \\
& +C_{1}\left(v_{(n+1) 2}(t)-v_{(n-1) 2}(t)\right) v_{n 0}(t) \\
& +C_{1}\left(v_{(n+1) 0}(t)-v_{(n-1) 0}(t)\right) v_{n 2}(t) \\
& +C_{1}\left(v_{(n+1) 1}(t)-v_{(n-1) 1}(t)\right) v_{n 1}(t) \\
& +C_{2}\left(v_{(n+1) 0}(t)-v_{(n-1) 0}(t)\right) v_{n 1}(t) \\
& +C_{1}\left(v_{(n+1) 2}(t)-v_{(n-1) 2}(t)\right) v_{n 0}(t) \\
& +C_{2}\left(v_{(n+1) 1}-v_{(n-1) 1}\right) v_{n 0}(n, t) \\
& +C_{3}\left(v_{(n+1) 0}(n, t)-v_{(n-1) 0}(n, t)\right) v_{n 0}(t),
\end{aligned}
$$

with

$$
\begin{gathered}
v_{n 3}(0)=0, \\
v_{n 3}(t)=6 n t\left(C_{2}+C_{3}+2 C_{1}^{2}(1+6 t)\right. \\
\left.+C_{1}^{3}(1+6 t)^{2}+C_{1}\left(1+2 C_{2}(1+6 t)\right)\right) .
\end{gathered}
$$

Adding (49), (52), (55), and (58), we obtain

$$
\begin{aligned}
v_{n}(t)= & v_{n 0}(t)+v_{n 1}\left(t, C_{1}\right)+v_{n 2}\left(t, C_{1}, C_{2}\right) \\
& +v_{n 3}\left(t, C_{1}, C_{2}, C_{3}\right), \\
v_{n 3}(t)= & n+6 C_{1} n t+6 n t\left(C_{1}+C_{2}+C_{1}^{2}(1+6 t)\right) \\
& +6 n t\left(C_{2}+C_{3}+2 C_{1}^{2}(1+6 t)+C_{1}^{3}(1+6 t)^{2}\right. \\
& \left.+C_{1}^{3}(1+6 t)^{2}+C_{1}\left(1+2 C_{2}(1+6 t)\right)\right) .
\end{aligned}
$$

For the computation of the constants $C_{1}, C_{2}$, and $C_{3}$ applying the method of least square mentioned in (16)-(18), we get

$$
\begin{aligned}
& C_{1}=-0.23640805995815892, \\
& C_{2}=-0.039496242739004025, \\
& C_{3}=-0.012135699742215487 \text { for } n=1 .
\end{aligned}
$$

The exact solution of problem (42) is [9]

$$
v_{n}(t)=\frac{n}{1+6 t} .
$$

The HAM solution of (42) is given by [9]

$$
\begin{aligned}
v_{n}(t)= & (n+t)+h(n+1) t+3 h t^{2}+t h(1+h)(1+6 n) \\
& +\left(36 h^{2} n+6 h^{2}+3 h\right) t^{2}+18 h^{2} t^{3} .
\end{aligned}
$$

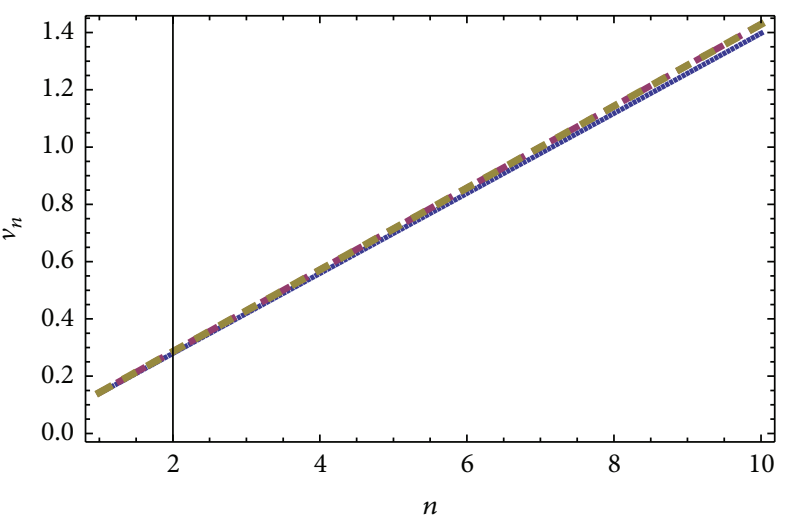

FIgUre 3: Comparisons of OHAM, HAM and exact solutions at $t=$ $1, h=-1$.

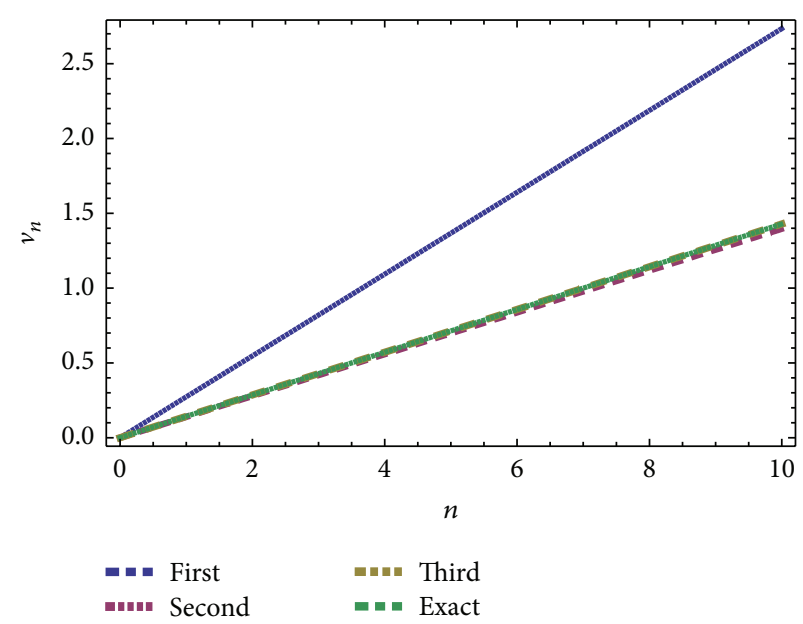

FIgure 4: Comparison of first, second, third of OHAM, HAM and exact solutions at $t=1, h=-1$.

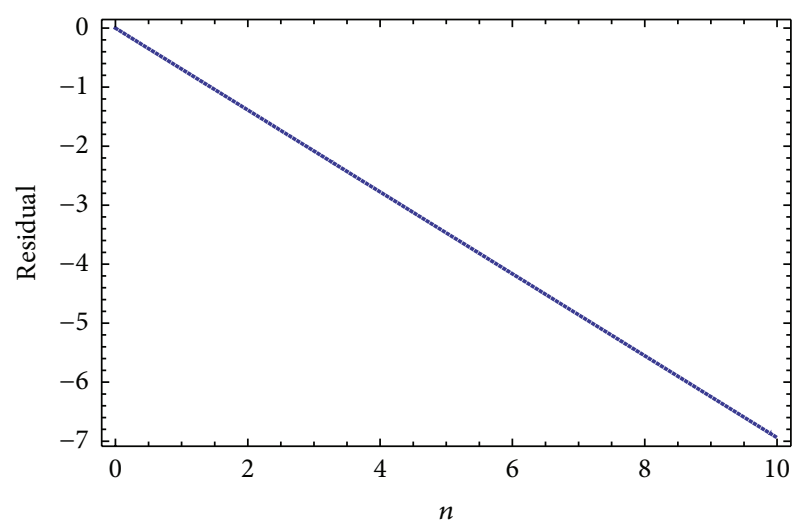

..... Residual

FIgURE 5: Residual of (42) at $t=1$. 


\section{Results and Discussions}

The formulation presented in Section 2 provides highly accurate solutions for the problems demonstrated in Section 3. We have used Mathematica 7 for most of our computational work. Table 1 and Figure 1 give the comparisons of OHAM with ADM and exact solutions for Model 1. Also the absolute errors at different values of $n$ at $t=1$ are given (Figure 4). Figure 3 shows the comparisons of OHAM results with HAM, exact at different values of $n$ at $t=1$ for Model 2. The convergence of approximate orders to exact solutions is given in Table 2 for Model 1 and Figure 5 for Model 2. The residuals have been plotted for Model 1 in Figure 2 and for Model 2 in Figure 5. We have concluded that the results obtained by OHAM are identical to the results obtained by ADM, HAM, and exact. OHAM converges rapidly with increasing of the order of approximation.

\section{Conclusion}

In this work, we have seen the effectiveness of OHAM [12-16] to differential-difference equations. By applying the basic idea of OHAM to differential-difference equations, we found it simpler in applicability, more convenient to control convergence, and involved less computational overhead. Therefore, OHAM shows its validity and great potential for the differential-difference equations arising in science and engineering.

\section{Conflict of Interests}

The authors declare that there is no conflict of interests regarding the publication of this paper.

\section{Acknowledgment}

The authors are thankful to the anonymous reviewers for their constructive and valuable suggestions.

\section{References}

[1] D. Levi and R. Yamilov, "Conditions for the existence of higher symmetries of evolutionary equations on the lattice," Journal of Mathematical Physics, vol. 38, no. 12, pp. 6648-6674, 1997.

[2] R. I. Yamilov, "Construction scheme for discrete Miura transformations," Journal of Physics A: Mathematical and General, vol. 27, no. 20, pp. 6839-6851, 1994.

[3] I. Yu. Cherdantsev and R. I. Yamilov, "Master symmetries for differential-difference equations of the Volterra type," Physica D: Nonlinear Phenomena, vol. 87, no. 1-4, pp. 140-144, 1995.

[4] A. B. Shabat and R. I. Yamilov, "Symmetries of nonlinear lattices," Leningrad Mathematical Journal, vol. 2, p. 377, 1991.

[5] D.-J. Zhang, "Singular solutions in Casoratian form for two differential-difference equations," Chaos, Solitons and Fractals, vol. 23, no. 4, pp. 1333-1350, 2005.

[6] K. Narita, "Soliton solution for a highly nonlinear differencedifferential equation," Chaos, Solitons and Fractals, vol. 3, no. 3, pp. 279-283, 1993.
[7] Z. Wang and H.-Q. Zhang, "New exact solutions to some difference differential equations," Chinese Physics, vol. 15, no. 10, pp. 2210-2215, 2006.

[8] W. Zhen and H. Zhang, "A symbolic computational method for constructing exact solutions to difference-differential equations," Applied Mathematics and Computation, vol. 178, no. 2, pp. 431-440, 2006.

[9] L. Zou, Z. Zong, and G. H. Dong, "Generalizing homotopy analysis method to solve Lotka-Volterra equation," Computers \& Mathematics with Applications, vol. 56, no. 9, pp. 2289-2293, 2008.

[10] Z. Wang, L. Zou, and H. Zhang, "Applying homotopy analysis method for solving differential-difference equation," Physics Letters A: General, Atomic and Solid State Physics, vol. 369, no. 1-2, pp. 77-84, 2007.

[11] Z. Wang, L. Zou, and Z. Zong, "Adomian decomposition and Padé approximate for solving differential-difference equation," Applied Mathematics and Computation, vol. 218, no. 4, pp. 13711378, 2011.

[12] N. Herişanu and V. Marinca, "Explicit analytical approximation to large-amplitude non-linear oscillations of a uniform cantilever beam carrying an intermediate lumped mass and rotary inertia," Meccanica, vol. 45, no. 6, pp. 847-855, 2010.

[13] V. Marinca and N. Herişanu, "Application of optimal homotopy asymptotic method for solving nonlinear equations arising in heat transfer," International Communications in Heat and Mass Transfer, vol. 35, no. 6, pp. 710-715, 2008.

[14] V. Marinca, N. Herişanu, C. Bota, and B. Marinca, "An optimal homotopy asymptotic method applied to the steady flow of a fourth-grade fluid past a porous plate," Applied Mathematics Letters, vol. 22, no. 2, pp. 245-251, 2009.

[15] V. Marinca, N. Herisanu, and I. Nemes, "A new analytic approach to nonlinear vibration of an electrical machine," Proceedings of the Romanian Academy, vol. 9, pp. 229-236, 2008.

[16] V. Marinca and N. Herişanu, "Determination of periodic solutions for the motion of a particle on a rotating parabola by means of the optimal homotopy asymptotic method," Journal of Sound and Vibration, vol. 329, no. 9, pp. 1450-1459, 2010.

[17] M. Idrees, S. Islam, and A. M. Tirmizi, "Application of optimal homotopy asymptotic method of the Korteqag-de-Varies equation," Computers \& Mathematics with Applications, vol. 63, pp. 695-707, 2012.

[18] H. Ullah, S. Islam, M. Idrees, and M. Arif, "Solution of boundary layer problems with heat transfer by optimal homotopy asymptotic method," Abstract and Applied Analysis, vol. 2013, Article ID 324869, 10 pages, 2013.

[19] R. Nawaz, H. Ullah, S. Islam, and M. Idrees, "Application of optimal homotopy asymptotic method to Burger equations," Journal of Applied Mathematics, vol. 2013, Article ID 387478, 8 pages, 2013.

[20] H. Ullah, S. Islam, M. Idrees, and R. Nawaz, "Application of optimal homotopy asymptotic method to doubly wave solutions of the coupled Drinfel'd-Sokolov-Wilson Equations," Mathematical Problems in Engineering, vol. 2013, Article ID 362816, 8 pages, 2013. 


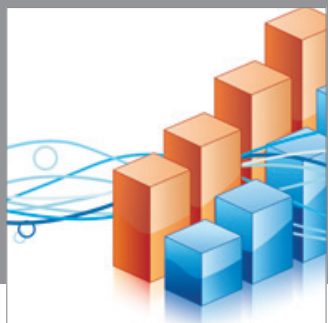

Advances in

Operations Research

mansans

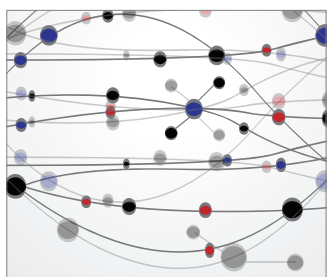

The Scientific World Journal
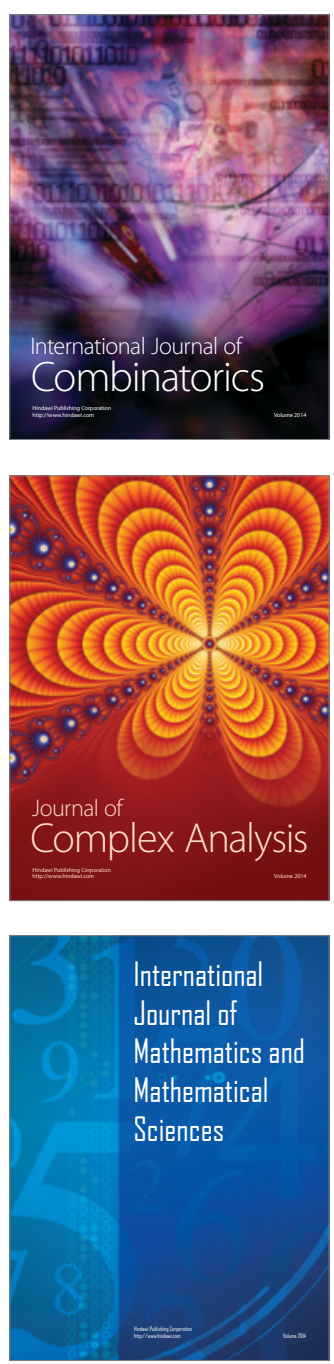
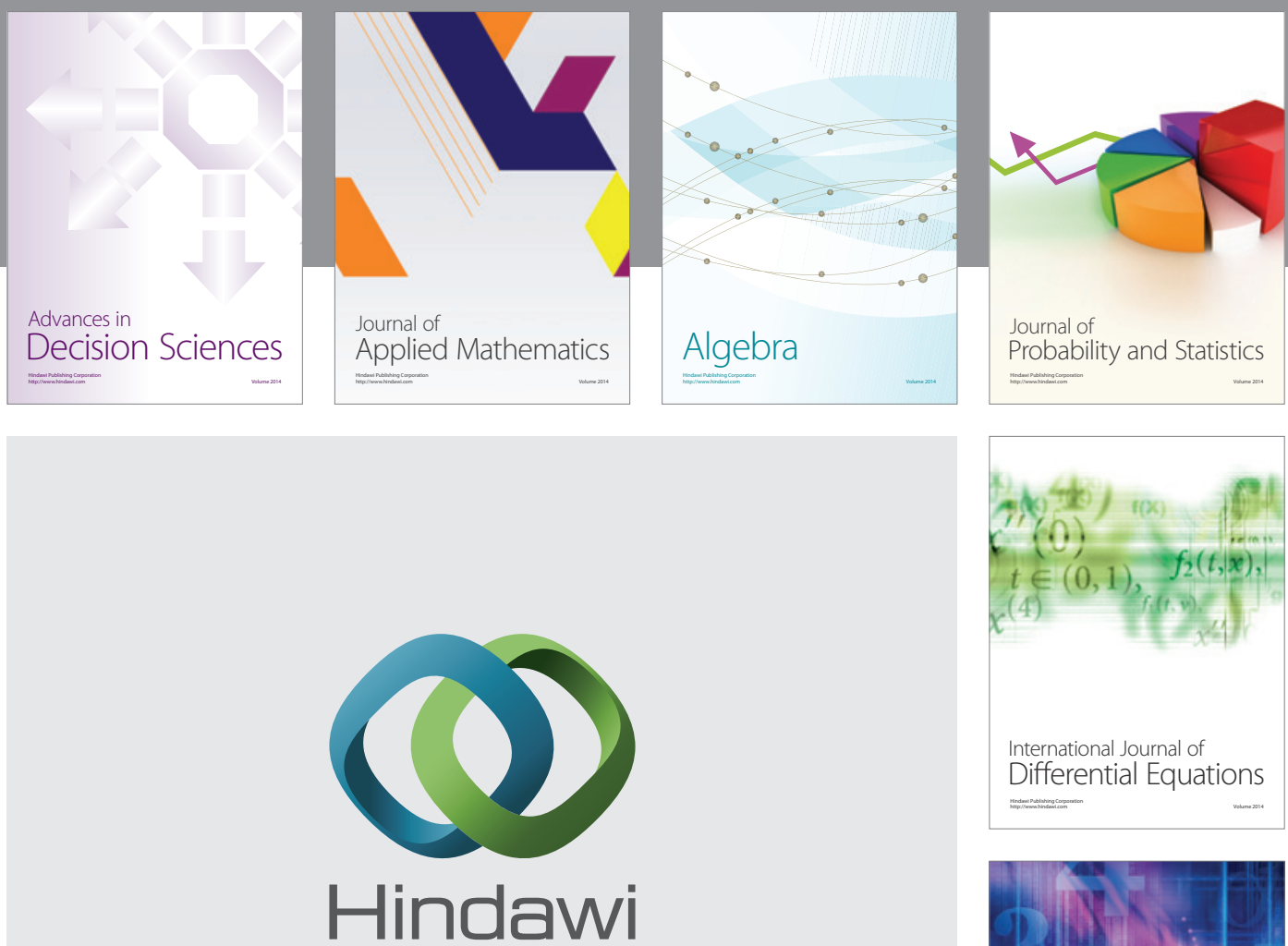

Submit your manuscripts at http://www.hindawi.com
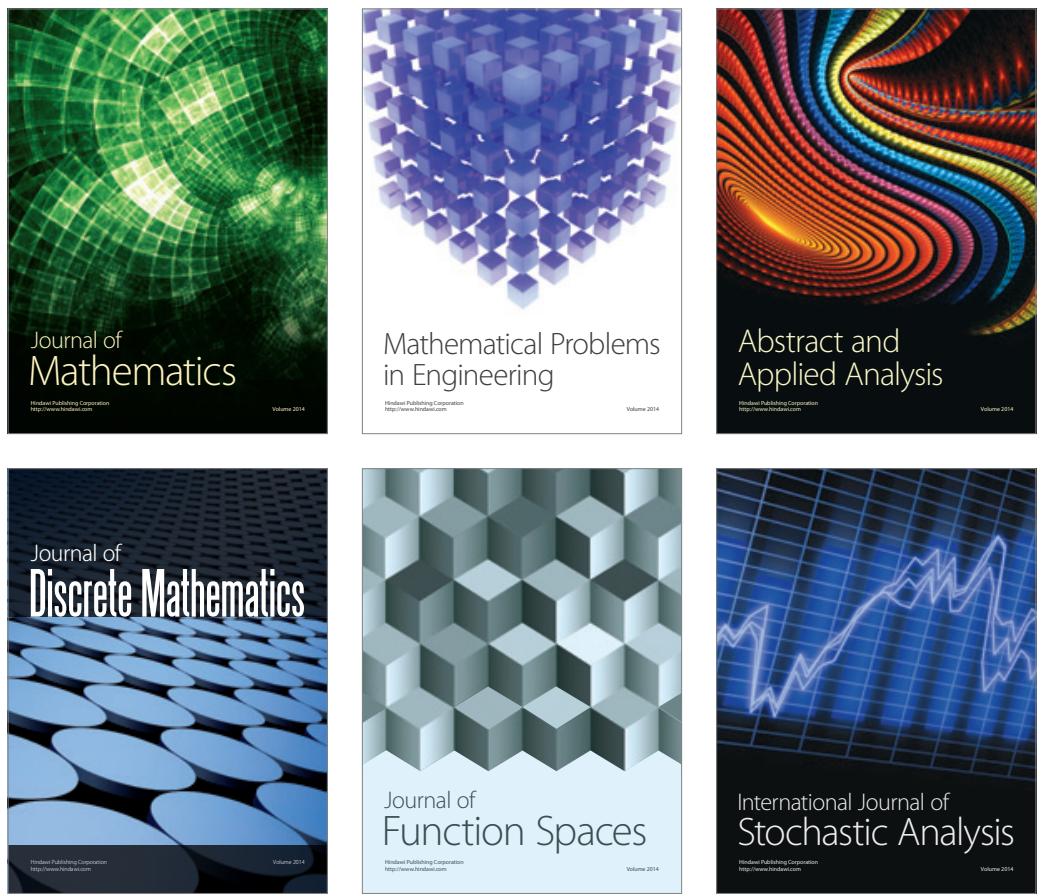

Journal of

Function Spaces

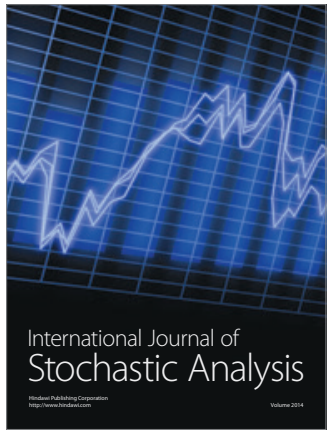

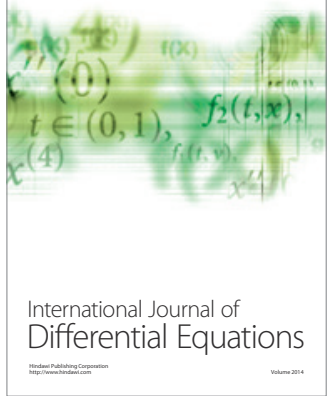
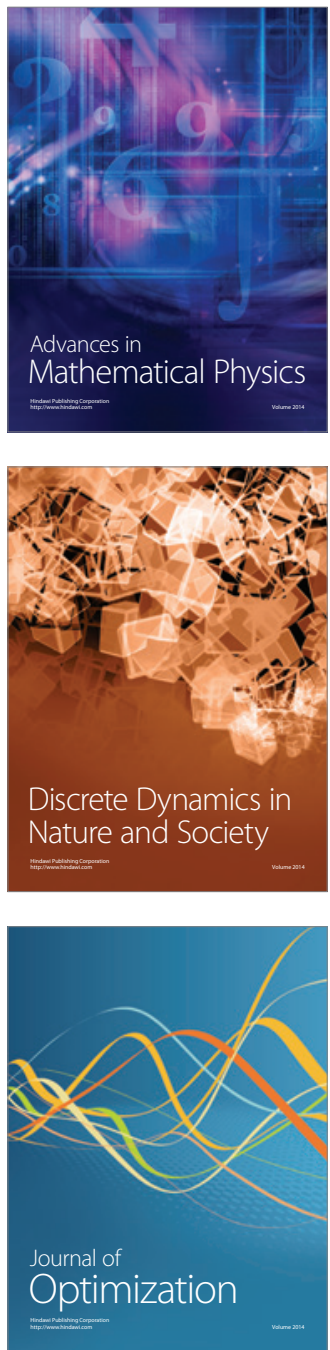